\title{
Study and Survey on Image Compression Techniques
}

\author{
G. Ramya' ${ }^{1}$ and K. N. Abdul Kader Nihal ${ }^{2}$ \\ ${ }^{1}$ Research Scholar, ${ }^{2}$ Assistant Professor \\ ${ }^{1 \& 2}$ Department of Computer Science, Jamal Mohamed College, Tiruchirappalli, Tamil Nadu, India \\ E-Mail: rajisramya@gmail.com, akn@jmc.edu
}

\begin{abstract}
In the Modern World, image is the important source for new technologies. The image compression is one of the important techniques which play a vital role in many applications. This paper presents the study of various image compression techniques which are used in the different Image Processing Applications. The advancement in the computer vision increases the demand of high data transmission speed also there is a need of large storage space. So, the image compression field is a necessary one for such applications where high volume of data is required to store and transmit. This paper focuses on the area of image compression, advantages of image compression, types of image compression and its different types of techniques. In addition to that, one of the new lossless image compression techniques $A B O$ is explained. In this paper, it gives the overview of the ABO Framework and the ABO mechanism.
\end{abstract}

Keywords: Image Compression, Adaptive Binary Optimization (ABO), Repetition and Correlation Coding (RCC)

\section{INTRODUCTION}

A. Image: An Image is a visual representation of anything. Based on human visual system, the images are defined as two-dimensional signal processed. The Image is undergoing some process to convert the analog signals to the digital form and then it's stored and transmits for various purposes. Image is easy to understandable and easy to access. An Image is a collection of pixels, and each pixel has a value. Now-a-days the technologies rule the world, in that new technology the images place a vital role in it. Visualizing any object is much easier to observe, searchable, and easy to access, it also helps to discover new ideas for efficient storage and fast transmission of image data [1].

B. Types of Images: Generally, there are four types of digital images. They are described below.

1. Binary Image: Binary images are the simplest type of images and it can take either 0 or 1 respectively for black and white. A binary image is considered as a 1bit image because it takes only one binary digit to represent each pixel. These types of images are mostly used in the fields in which the information is required only for general shape or outline. For example, Optical Character Recognition (OCR).

2. Gray-Scale Image: Gray-scale images are considered as a monochrome (one-color) images. They do not contain color information, but contain gray-level information only. The different gray levels are available based on number of bits used for each pixel. The typical grayscale images contain 8-bit data which allow us to have 356 different gray levels.

3. Colour Image: Colour images can be designed as a three band monochrome image data, in which each band of data correspond to a different color. The original information stored in the digital image data is the gray-level information in each spectral band. Color images are generally represented as Red, Green and Blue (RGB-images). Using the 8-bit monochrome image data as a model, the respective color image has 24-bit (pixel).

4. Multispectral Image: Multi-spectral images are the images of data which are not directly visible to human vision. This may include X-ray, infrared, acoustic, ultraviolet or radar data. This information is often represented in the visual form by mapping the different spectral bands to RGB components [2].

Table I list the different types of image formats and its features.

TABLE I IMAGE FoRmats AND ITS FeAtures

\begin{tabular}{|c|l|l|}
\hline S. No. & \multicolumn{1}{|c|}{ Format } & \multicolumn{1}{c|}{ Features } \\
\hline 1 & $\begin{array}{l}\text { GIF (Graphics } \\
\text { Interchange } \\
\text { Format) }\end{array}$ & $\begin{array}{l}\text { It consists of grayscale images, } \\
\text { black and white images. It states } \\
\text { logos, simple graphics and cartoon } \\
\text { style images. }\end{array}$ \\
\hline 2 & $\begin{array}{l}\text { JPEG (Joint } \\
\text { Photographic } \\
\text { Expert Group) }\end{array}$ & $\begin{array}{l}\text { It supports 8 bits grayscale image } \\
\text { and 24 bits colour images. It } \\
\text { provides the compression of } \\
\text { photographs and video stills. }\end{array}$ \\
\hline 3 & $\begin{array}{l}\text { TIFF (Tagged } \\
\text { Image File } \\
\text { Format) }\end{array}$ & $\begin{array}{l}\text { It is a flexible format. It saves 8 } \\
\text { or16 bits per colour in RGB. So } \\
\text { totally 24 or 48 bits. }\end{array}$ \\
\hline 4 & $\begin{array}{l}\text { BMP (Bitmap) } \\
\text { Microsoft window operating } \\
\text { system. BMP images are binary } \\
\text { files. }\end{array}$ \\
\hline 5 & $\begin{array}{l}\text { PNG (Portable } \\
\text { Network }\end{array}$ & $\begin{array}{l}\text { It has smaller size and more colors } \\
\text { when compared to other formats }\end{array}$ \\
\hline 6 & RAW & $\begin{array}{l}\text { It has a file size smaller than the } \\
\text { TIFF format. It is available on } \\
\text { digital cameras. }\end{array}$ \\
\hline
\end{tabular}


C. Image Compression: Image compression is the process of reducing the irrelevance and redundancy data in the image. This compression is mainly used to store and transmit the data in an efficient form. Compression is achieved by the removal of any one among the three basic data redundancies. They are:

1. Coding Redundancy,

2. Inter-Pixel Redundancy,

3. Psycho Visual Redundancy.

The Fig.1 explains the Process of image compression and decompression.

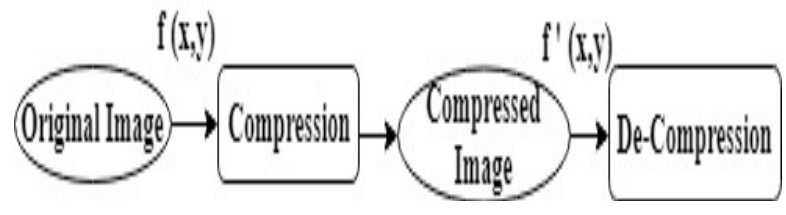

Fig.1 Compression and Decompression Process

In the Reconstructed (decompressed) image,

Where,

$$
f^{\prime}(x, y)=f(x, y)+e(x, y)
$$

$\hookrightarrow \mathrm{f}(\mathrm{x}, \mathrm{y})$ is the original image,

$\hookrightarrow \mathrm{e}(\mathrm{x}, \mathrm{y})$ is the noise or error after the compression.

\section{Advantages of Compression}

1. Size Reduction:The most significant benefit of the image compression is the size reduction. Unless the user edits the physical size of the image, it retains the same size of the original image. Only the storage space on the hard drive is reduced.

2. Slow Devices:In some devices the large size images may load slowly. But the Image compression helps to load the large size images faster even in the slower devices[3].

\section{IMAGE COMPRESSION TECHNIQUES}

There are different types of Image compression techniques. They are.

1. Lossless Compression

2. Lossy Compression

The Fig.2 represents the two types of image compression techniques.

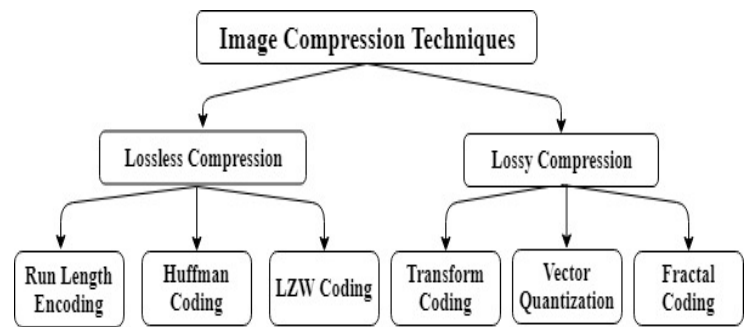

Fig.2 Types of Image Compression Techniques

\section{A. Lossless Compression Technique}

In the Lossless compression Technique, the original image can get back perfectly (without any loss of data) from the compressed image. This Method is also known as noiseless method because it does not add any noise to the image.Some methods which are included in the lossless compression techniques are:

1. Run length Encoding

2. Huffman Encoding

3. LZW Coding.

\section{B. Lossy Compression Technique}

In this Lossy Compression Technique, the original image cannot get back from the compressed image, but similarly related to it. This method provides much higher compression ratio when compared to the lossless compression method. The major performance factors of a lossy compression scheme include the following.

1. Compress Ratio

2. Signal to Noise Ratio

3. Speed of Encoding and Decoding.

Methods which are included in the lossy compression techniques are.

1. Transform Coding

2. Vector Quantization

3. Fractal Quantization

\section{Lossless Compression Techniques}

\section{Run Length Encoding}

This is a very simple form of lossless image compression method used for sequential data and for the repetitive data. Instead of storing the original runs of data it stores the single data value and its number of counts. In this method, it replaces the sequences of identical symbol(pixels) called runs. For a gray scale images the run length code is represented by a sequence of $\mathrm{Vi}$, $\mathrm{Ri}$ Where $\mathrm{Vi}$ is the intensity of the pixel and $\mathrm{Ri}$ refers to the number of consecutive pixels with the intensity $\mathrm{Vi}$ as shown in the figure. It is most useful for simple graphic images such as line drawings, icons and animations. And, it is not useful with the files that don't have many runs as it could greatly increase the file size. This technique is used in fax machines [4]. For Example, Table II depicts the sequential data of an original image.

TABle II Sequential Data Of An IMAge

\begin{tabular}{|l|l|l|l|l|l|l|l|l|l|}
\hline $\mathbf{7 3}$ & $\mathbf{7 3}$ & $\mathbf{7 3}$ & $\mathbf{1 0}$ & $\mathbf{1 0}$ & $\mathbf{1 0}$ & $\mathbf{1 0}$ & $\mathbf{3 7}$ & $\mathbf{3 7}$ & $\mathbf{3 7}$ \\
\hline
\end{tabular}

Table III shows the result of compression using RLE technique.

TABLE III COMPRESSED DATA OF AN ORIGINAL IMAgE

\begin{tabular}{|l|l|l|}
\hline 73,3$\}$ & $\{10,4\}$ & $\{37,3\}$ \\
\hline
\end{tabular}




\section{Huffman Coding}

Huffman Coding is one of the lossless image compression techniques. It is proposed by Dr. David A. Huffman in the year 1952. He proposed this method for the construction of the minimum redundancy code [5].This is the general method for coding symbols based on their statistical occurrence of frequencies (probability). Each pixel in the images is considered as a symbol. The smaller numbers of bits are assigned to the symbols that occur more frequently, and larger numbers of bits are assigned to those symbols which are occurring less frequently. In this Huffman code is a prefix code. Which means that the binary code of any symbol is not the prefix code of any other symbol? In early stages of compression, the lossy methods are mostly used for image coding standards then use the Huffman coding as the final step [6].

Example for Huffman Coding is explained in step-by-step

Step 1: Enter the string as input

\begin{tabular}{|c|c|c|c|c|}
\hline 22 & 1 & 3 & 7 & 10 \\
\hline G & $\mathbf{P}$ & $\mathbf{R}$ & $\mathbf{L}$ & $\mathbf{S}$ \\
\hline
\end{tabular}

Step 2: Sort the given data based on the frequencies

\begin{tabular}{|c|c|c|c|c|}
\hline 1 & 3 & 7 & 10 & 22 \\
\hline $\mathbf{P}$ & $\mathbf{R}$ & $\mathbf{L}$ & $\mathbf{S}$ & G \\
\hline
\end{tabular}

Step 3: Select the least two frequencies count.

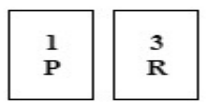

Step 4: Merge the two selected frequencies together with their sum and update the data.

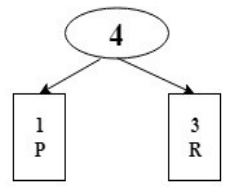

Step 5: Repeat the steps 2,3 and 4 until all the codes are processed.

Finally, Fig.3 shows the Huffman Tree for the given example:

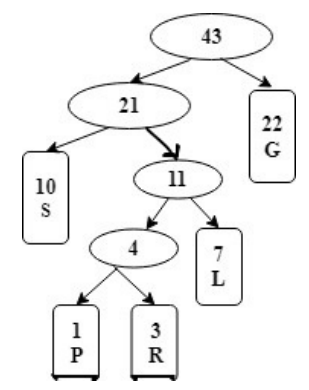

Fig.3 Huffman tree for given code

\section{LZW Coding}

Lempel-Ziv-Welch is the full form of this LZW coding. It is the name of the three persons who created this universal lossless data compression algorithm. They names are
Abraham Lempel, Jacob Ziv, and Terry Welch. First, it was published by Lempel and Ziv in 1978 and in 1984 welch improved it as an implementation of LZ78 algorithm. This is a dictionary-based coding. It can be static or dynamic. In the static dictionary coding, the dictionary is fixed but, In the dynamic dictionary coding, the dictionary is updated. LZW compression technique is widely used for GIF image format and for the UNIX files [7].

\section{Lossy Compression Techniques}

\section{Transform Coding}

It is one of the lossy compression techniques which are mostly used for natural data like photographic images. This coding is used to convert the spatial image pixel values to transform coefficient values. In this process, the number of produced coefficients value is equal to the number of pixels transformed. There are various types of transforms which are used for picture coding such as Walsh-Hadamard, Fourier, lapped orthogonal, Karhonen-Loeve, Discrete Cosine (DCT)[8]

\section{Vector Quantization}

Vector quantization is developed based on the principle of scalar quantization. In this, the normal entropy coding is used. There are two different methods linear and non-linear. Among this one method is selected dependents on the component of a vector. Using this technique, a dictionary of fixed-size vectors called code vectors is generated. The image is first partitioned into blocks of image vectors. And then for each image vector, the nearest matching vector in the dictionary is determined and its index is used as the encoding of the original image vector [9].

\section{Fractal Coding}

Fractal coding is the one lossy compression technique. In this technique, it first divides the image into segments by using some common points such as edges of the image, texture color difference and frequency. Generally, the one parts of an image and the other parts of the images usually resemble. Here, the dictionary is used as a look up table for the fractal segments. And the library contains the codes which are the suitable set of numbers. By performing this algorithm operation, the fractals are operated and finally the image is encoded [10].

\section{ADAPTIVE BINARY OPTIMIZATION (ABO) TECHNIQUE}

\section{A. Framework of $A B O$}

Adaptive Binary Optimization is a lossless image compression algorithm by Matrix View. ABO technique uses the right method to compress the high correlation in the digital signals and additional compression with standard encoding methods such as Huffman coding. It is a new way of transforming the data unlike JPEG, TIFF, JPEGLS, PNG, GIF, JPEG2000, MP3, MPEG1, MPEG2 or MPEG4. ABO 
does not depend on the complex transforms and the elimination of the data to achieve compression like other traditional systems.It works based on the unique breakthrough process called Repetition and Correlation Coding (RCC). It exploits the repetition and correlation found in data. This establishes a strong foundation for the additional modelling and the source coding of data and it results in a revolutionary data transformation. At every level of the network, the effects of this bottom-up transformation are very strong. The amazing $\mathrm{ABO}$ technique just transforms the way of applied, stored and retrieved the data with high compressibility in addition to that; it compresses the image without any loss of data and integrity.

\section{RCC Construction}

RCC is a lossless compression technique. Also, it is a pixelto-pixel lossless compression with zero Means Square Error (MSE). It achieves a very high level of compression based on the repetitions of coding. Through the modification of the generic RCC method, the RCC with encryption support method is developed. The framework of generic RCC method is formed by the encryption modules. In the RCC, there are two types of RCC methods. One is RCC Horizontal and another one is RCC Vertical. In RCC Horizontal transformation the only one bit-plane is used to code the repetition values and it encoded in the horizontal direction only. The RCC Vertical transformation is same as the RCC Horizontal transformation but, in this the image data is compared in a non-raster order.

\section{Bit Plane}

Bit plane is a table which index with the array of zeros and ones. The bit plane increases the ratio of the compression without any loss or increase in the data set. In the bit plane indexing process, the original image data is converted to various types of bit planes. A bit plane of ones and zeros is obtained along with the index of the image. Then the restoration of the original image is perfectly lossless with the index and the bit plane. The selection of the bit plane is based on the application or final product. In the RCC method, each and every element is compared with its previous element. If both of them are equal then the value " 1 " is stored in the bit-plane Otherwise, the value " 0 " is stored in it.

\section{Data Value Stored}

The data sequence with only bit plane value 0 is stored in data value thus discard the redundant value [11].

Table IV represent the data sequence of original image and its bit-plane value and final value stored in the data store.

Table IV Shows The Data Sequence, Bit-Plane And Value Stored

\begin{tabular}{|l|c|c|c|c|c|c|c|c|}
\hline Data Sequence & 31 & 31 & 31 & 46 & 46 & 73 & 73 & 73 \\
\hline Bit Plane & 0 & 1 & 1 & 0 & 1 & 0 & 1 & 1 \\
\hline Value Stored & 31 & & & 46 & & 73 & & \\
\hline
\end{tabular}

\section{B. ABO Mechanism}

Adaptive Binary Optimization (ABO) is the process of compressing the highly correlated image data. This technique is based on the Repetition and Correlation Coding (RCC) Method. It first captures the image and then it converts it into the digital form to reshape and encode the image data then finally it results in stores and retrieve the compressed data. Thesteps which are involved in the ABO Mechanism are listed below:

1. Image Acquisition

2. Digital Transformation

3. Encoding

4. Value Store.

1. Image Acquisition: Image capturing is the first step in the image processing. The image is captured by a camera and converted into a manageable entity. This process is known as Image Acquisition.For example, one captured image is given below:

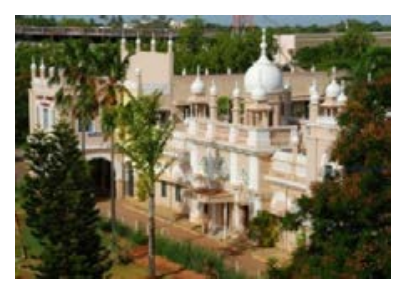

Fig.4 Image Acquisition

2. Digital Transformation: The next step is to converting the image into the digital form to obtain the digital data. A digital image is a representation of two-dimensional set of image data. It reshaping the digital data into a digital data matrix of image data values is carried out.

Example: The captured image is transformed to the digital transformation. Table $\mathrm{V}$ shows the converted pixel value (digital value) of a captured image.

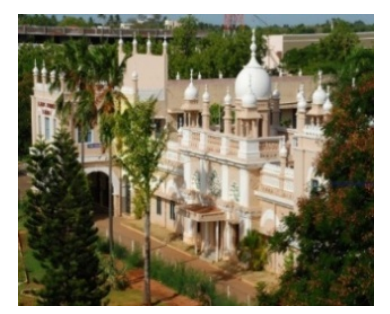

Fig.5 Captured Image

TABLe V PiXel VALUes

\begin{tabular}{|c|c|c|c|c|}
\hline \multicolumn{5}{|c|}{ Pixel Value of the image } \\
\hline 100 & 100 & 100 & 120 & 120 \\
\hline 130 & 130 & 130 & 170 & 170 \\
\hline 50 & 50 & 90 & 90 & 90 \\
\hline 30 & 30 & 30 & 150 & 150 \\
\hline 70 & 70 & 70 & 110 & 110 \\
\hline
\end{tabular}


3. Encoding: In this process, the redundant pixel value of an image which is in the form of digital data matrix is converted into a bit plane index value and further it processed to form the compressed data. The rules to encoding the pixel value of an image is: -

a. If the pixel value is equal to its adjacent pixel value, then the bit plane is recorded as 1 .

b. Otherwise, the bit plane recorded as 0 .

Example, Table VI shows the encoded values of an original image are stored in the bit-plane.

TABLe Vi EnCOded Bit-Plane V
\begin{tabular}{|c|c|c|c|c|}
\hline \multicolumn{5}{|c|}{ Bit-Plane } \\
\hline 0 & 1 & 1 & 0 & 1 \\
\hline 0 & 1 & 1 & 0 & 1 \\
\hline 0 & 1 & 0 & 1 & 1 \\
\hline 0 & 1 & 1 & 0 & 1 \\
\hline 0 & 1 & 1 & 0 & 1 \\
\hline
\end{tabular}

4. Value Store: In final step, the storage of compressed data is carried out where in each pixel value is compared with the previous pixel value [12].

If (Current pixel $=$ Previous pixel)

Record the previous value

else

Record the current value.

Example:Table VII depicts the encoded final value of an original image.

TABLE Vil It Store The EnCoded VALUes

\begin{tabular}{|c|c|c|}
\hline \multicolumn{3}{|c|}{ Value Stored } \\
\hline 100 & 120 & 130 \\
\hline 170 & 50 & 90 \\
\hline 30 & 150 & 70 \\
\hline 110 & - & - \\
\hline
\end{tabular}

\section{CONCLUSION}

This paper presents various techniques of image compression. This paper explains about three different techniques of lossless compression. They are Run Length Encoding, Huffman Coding, LZW Coding. The RLE (Run
Length Encoding) technique has mostly suitable for the gray-scale images only. In the Huffman coding, the compression of image files that contain long runs of identical pixels, which is not so efficient when compared to others. And in the LZW Coding, there is a need to use the dictionary for encoding process; it requires a lot of space. To overcome all these issues, the Adaptive Binary Optimization (ABO) technique is used for the lossless compression. This technique is comparatively best with other lossless compression methods.

\section{REFERENCES}

[1] K.A.K. Nihal and A.M. Shanavas, "A Survey and Study of Image Compression Methods,"IOSR Journal of Computer Engineering, Vol.16, No.4, pp. 11-16, August 2014.

[2] Lecture2, uotechnology, [Online] Available at:http://uotechnology .edu.iq/ce/lecture\%202013n/4th\%20Image\%20Processing\%20_Lectu res/DIP_Lecture2.pdf. Accessed 22 January 2019.

[3] A. Kaur and J. Singh, "Review on Image Compression Techniques and Advantages of Image Compression”, International Journal of Advance Research in Science and Engineering, Vol.5, August 2016.

[4] K. Kapoor and H. Kaushik, "Review Paper on Various Image Compression Techniques”, International Journal of All Research Education and Scientific Methods, Vol. 6, No. 7, pp. 30-35, July 2018.

[5] F.I. Khandwani and P.E. Ajmire, "A Survey of Lossless Image Compression Techniques", International Journal of Electrical Electronics and Computer Science Engineering, Vol. 5, No.1,pp. 3942, February 2018.

[6] A.K. Singh and A.K. Malviya, "A Survey on Image Compression Methods", International Journal of Engineering and Computer Science, Vol. 6, No. 5, pp. 21393-21400, May 2017.

[7] G. Vijayvargiya, D.S. Silakari and D. Pandey, "A Survey: Various Techniques of Image Compression”, International Journal of Computer Science and Information Security, Vol. 11, No. 10, October 2013.

[8] A.B. Kaimal, S. Manimurugan and C. Devadass, "Image Compression Techniques: A Survey", International Journal of Engineering Inventions, Vol. 2, No. 4, pp. 26-28, February 2013.

[9] P.B. Pokle and N.G. Bawane, "Comparative Study of Various Image Compression Techniques", International Journal of Scientific and Engineering Research, Vol. 4, No. 5, pp. 1902-1908, May 2013.

[10] D.V. Rojatkar, N.D. Borkar, B.R. Naik and R.N. Peddiwar, "Image Compression Techniques: Lossy and Lossless”, International Journal of Engineering Research and General Science, Vol. 3, No. 2, pp. 912917, April 2015.

[11] A.R.M. Shanavas and M. Shameera, "Secured Lossless Medical Image Compression Based On Adaptive Binary Optimization”, IOSR Journal of Computer Engineering, Vol. 16, No. 2, pp. 43-47, April 2014.

[12] M. Shameera and A.R.M. Shanavas, "Analysis of Adaptive Binary Optimization for Lossless Medical Image Compression”, International Journal of Engineering Research and Technology, Vol. 3, No. 7, pp. 1221-1224, July 2014. 